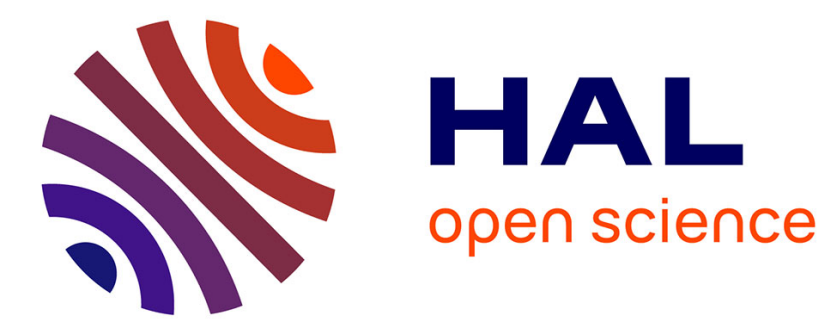

\title{
Schizophrénie intergénérationnelle
}

Bruno Decreuse, Bertrand Wigniolle

\section{To cite this version:}

Bruno Decreuse, Bertrand Wigniolle. Schizophrénie intergénérationnelle. Recherches Economiques de Louvain - Louvain economic review, 2006, 72 (1), pp.49-74. 10.3917/rel.721.0049 . halshs-00185314

\section{HAL Id: halshs-00185314 https://shs.hal.science/halshs-00185314}

Submitted on 5 Nov 2007

HAL is a multi-disciplinary open access archive for the deposit and dissemination of scientific research documents, whether they are published or not. The documents may come from teaching and research institutions in France or abroad, or from public or private research centers.
L'archive ouverte pluridisciplinaire HAL, est destinée au dépôt et à la diffusion de documents scientifiques de niveau recherche, publiés ou non, émanant des établissements d'enseignement et de recherche français ou étrangers, des laboratoires publics ou privés. 


\title{
Schizophrénie intergénérationnelle*
}

\author{
Bruno DECREUSE ${ }^{\dagger}$ \\ GREQAM, Université de la Méditerranée \\ Bertrand WIGNIOLLE ${ }^{\ddagger}$ \\ EUREQua, Université Paris I
}

Résumé: L'amour lorsqu'il est poussé à son paroxysme confine à l'étouffement. Telle est la morale de cet article dans lequel un parent altruiste module son offre de travail en partie pour assurer le meilleur niveau de vie possible à son enfant. Lorsque le travail des parents et celui des enfants sont parfaitement substituables dans la production, ce comportement pousse les salaires à la baisse. Si les salaires horaires sont rigides, l'emploi est rationné et les jeunes sont les premières victimes de ce rationnement. Nous montrons que ce mécanisme est à l'origine de fluctuations endogènes entre chômage et plein emploi lorsque la productivité des travailleurs âgés est négativement influencée par la durée de chômage passée. Nous analysons également le soutien politique pour le salaire minimum lorsque la productivité des jeunes est soumise à un aléa : le salaire minimum, l'offre de travail des vieux et le chômage des jeunes augmentent tous trois avec le facteur d'altruisme.

Abstract: Love pushed beyond its paroxysm leads to suffocation. That is the moral of this article, which depicts an altruistic parent setting her labour supply to offer the best living conditions to her child. When parents and children are perfectly substituable in production, this behaviour pushes all wages down. If hourly wages are rigid, employment is rationed and the youth are the primary victims of such rationing. We show this mechanism may originate endogenous fluctuations between under and full employment when the older workers' productivity negatively responds to past unemployment. We also analyse the political support for the minimum wage. When the youth productivity is random, the minimum wage, the older workers'

\footnotetext{
*Nous tenons à remercier deux rapporteurs de cette revue.

${ }^{\dagger}$ GREQAM - 2 rue de la charité, 13236 Marseille cedex 02. E-mail: decreuse@ehess.univ-mrs.fr

${ }_{\ddagger}^{\ddagger}$ EUREQua, Université Paris I Panthéon-Sorbonne - Maison des Sciences Economiques, 106-112 boulevard de l'Hôpital, 75647 Paris cedex 13, France. E-mail: wignioll@univ-paris1.fr
} 
labour supply and youth unemployment together rise with the intensity of parental altruism.

Classification J.E.L: D64; E24; J22

Mots-clés: Générations imbriquées; Altruisme; Offre de travail endogène; Chômage

Keywords: OLG model; Altruism; Endogenous labour supply; Unemployment 
"Qui veut faire l'ange fait la bête" Pascal, Pensées, Section VI : Les philosophes, Pensée 358

\section{Introduction}

De nombreux économistes, et parmi les plus prestigieux, ont consacré une partie importante de leurs réflexions à expliquer comment l'interaction de motivations parfaitement égoïstes pouvait conduire à un résultat collectivement souhaitable. Voici l'oeuvre de la Providence pour Boisguilbert, de la main invisible pour Smith, ou encore de l'ordre spontané pour Hayek. Ces auteurs ambitionnaient d'appréhender le fonctionnement de la société de manière globale. De ce point de vue, le présent travail adopte une perspective beaucoup plus modeste, puisqu'il ne considère qu'un aspect seulement des relations humaines. Il n'empêche qu'il propose un cas d'école, où des ressorts individuels altruistes se traduisent par une aggravation même de la situation économique de ceux et celles qui sont aimés.

L'amour dont il est question est l'amour filial, c'est-à-dire celui que la plupart des parents portent à leurs enfants. La situation de marché que nous abordons est celle du marché du travail, et en particulier les difficultés rencontrées par les générations les plus jeunes. La thèse que nous proposons est la suivante: le souci de fournir à leurs enfants un niveau de vie élevé pousse les travailleurs âgés à obtenir des revenus incompatibles avec le plein emploi, ce qui conduit à l'émergence du chômage des jeunes. En somme, l'altruisme intergénérationnel est quelque peu contre-productif puisqu'il contribue au rejet des jeunes travailleurs sur le marché de l'emploi.

Notre argument trouve son origine dans la coexistence de deux faits notoires, traduisant l'ambiguïté des relations intergénérationnelles sur le marché du travail.

D'une part, le fonctionnement institutionnel du marché du travail favorise les travailleurs plus âgés, et ce probablement au détriment des plus jeunes. La protection de l'emploi, le salaire minimum, voire le rôle des syndicats dans la fixation des salaires, procurent des rentes à ceux dont la situation est bien assise. Mais ces mêmes rentes se forment aux dépends des entrants, les outsiders ${ }^{1}$. Comme les travailleurs plus âgés constituent le gros du bataillon des travailleurs installés, et les travailleurs plus jeunes l'essentiel des entrants, il n'est pas absurde de penser que les jeunes "payent" en quelque sorte la situation économique enviable de leurs aînés. La figure 1 étaye cette intuition. Elle représente pour 8 pays de l'OCDE le ratio masculin du taux de chômage par classe d'âge au taux de chômage global. A l'exception notable de l'Allemagne, les travailleurs les plus jeunes sont bien plus exposés que les autres au risque chômage.

\footnotetext{
${ }^{1}$ Lindbeck et Snower (1988) constitue la référence obligée de la littérature insider/outsider.
} 


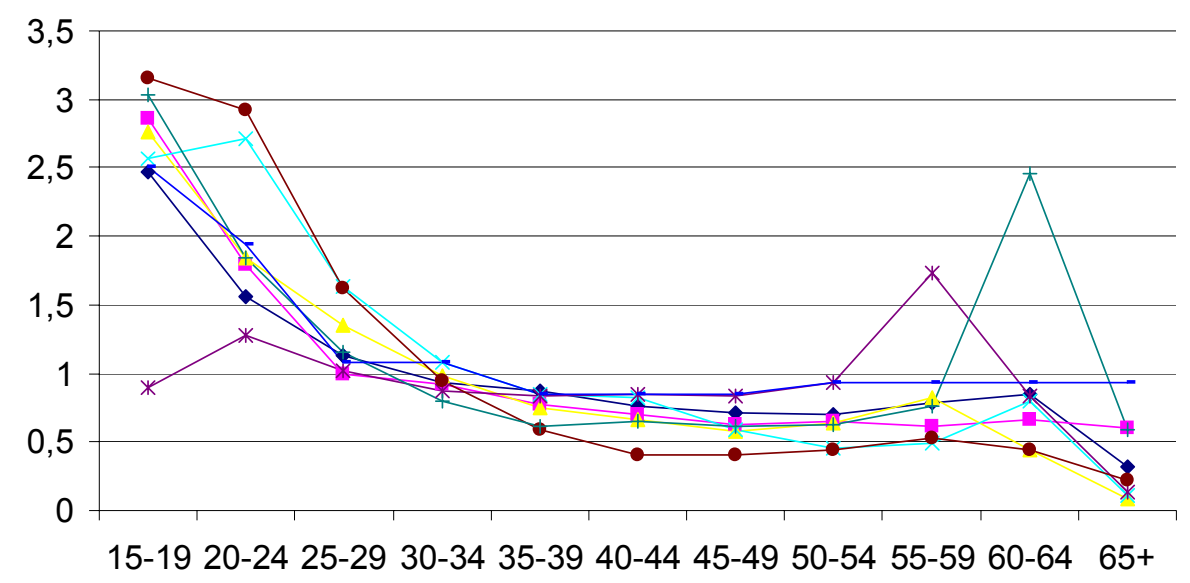

\begin{tabular}{lll}
$\rightarrow-$ Canada $\rightarrow$ USA & Espagne & $\rightarrow$ France \\
$\rightarrow$ Allemagne $\rightarrow$ Italie & $\leftarrow$ Japon $\quad-$ RU \\
\hline
\end{tabular}

Fig.1 : Ratio masculin du taux de chômage par classe d'âge au taux de chômage global dans 8 pays de l'OCDE, 1997

Source : ILO Statistics (2000)

Note : 1998 pour la France

D'autre part, les parents corrigent les conséquences de ces difficultés à l'aide de transferts financiers importants en direction de leurs enfants ${ }^{2}$. Ces transferts peuvent prendre un aspect concret, comme la délivrance d'un revenu complémentaire. Mais les transferts les plus visibles sont probablement liés au départ sans cesse retardé du foyer familial. Le Tableau 1 est tiré de Becker et al (2002). Il concerne les pays de l'Union Européenne, et montre que les départs les plus tardifs se produisent dans les pays méditerranéens, où justement le chômage des jeunes est le plus prégnant. Dans cette perspective, les travailleurs plus âgés sont particulièrement attentionnés à l'endroit des plus jeunes.

\footnotetext{
${ }^{2}$ Ce n'est, bien entendu, pas la seule motivation de ce type de transfert.
} 


\begin{tabular}{|l|l|l|}
\hline & $15-24$ & $25-29$ \\
\hline Belgique & 75 & 18 \\
\hline Danemark & 57 & 8 \\
\hline Allemagne & 73 & 21 \\
\hline Grèce & 82 & 49 \\
\hline Espagne & 90 & 60 \\
\hline France & 71 & 18 \\
\hline Irlande & 74 & 32 \\
\hline Italie & 90 & 56 \\
\hline Pays-Bas & 70 & 15 \\
\hline Autriche & 75 & 29 \\
\hline Portugal & 86 & 50 \\
\hline Finlande & 50 & 6 \\
\hline Suède & 60 & 5 \\
\hline Royaume-Uni & 67 & 18 \\
\hline UE & 76 & 30 \\
\hline
\end{tabular}

Tableau 1: Pourcentage de chaque groupe résidant dans le foyer parental en 1995.

Source: European Communities (1999), Tables B-15/16/20/21

Il est donc établi que les comportements parentaux expriment simultanément mépris et intérêt pour la situation économique des enfants. Bien entendu, l'incohérence de ces deux types de comportement n'est qu'apparente: c'est au travers d'un comportement collectif que les travailleurs âgés nuisent aux travailleurs plus jeunes, alors que les compensations financières évoquées prennent place au niveau familial. Nous renversons la perspective dans ce travail, en suggérant que les difficultés rencontrées par les travailleurs les plus jeunes sont justement liées à l'intensité de l'amour filial que leurs parents leur portent.

L'argument prend corps dans un modèle volontairement simple, que l'on peut caractériser en décrivant ses trois ensembles d'hypothèses spécifiques. Celles-ci affèrent, respectivement, aux conditions démographiques et techniques de la production, au cadre institutionnel du marché du travail, et aux relations intra-familiales.

Deux générations d'individus coexistent à chaque période sur le marché du travail, les "jeunes" et les "vieux". Les deux types de travail sont parfaitement substituables dans la production, et le rendement marginal du facteur travail est strictement décroissant. Ces deux dernières hypothèses garantissent que toute augmentation de la quantité de travail fournie par un groupe de travailleurs se traduit par une réduction de la productivité marginale du travail de tous. Les travailleurs âgés sont plus productifs, et peuvent moduler 
l'intensité de leur travail ${ }^{3}$. Les travailleurs jeunes ne le peuvent pas. Nous pouvons ainsi isoler les phénomènes uniquement attribuables au comportement des travailleurs âgés ${ }^{4}$.

Le cadre institutionnel du marché du travail est marqué par la présence d'un salaire minimum, et par une priorité à l'embauche des travailleurs âgés. Le salaire minimum a une signification autonome, mais il représente en sus l'ensemble des rigidités salariales susceptibles d'éloigner les rémunérations de la productivité marginale du travail. On songe ainsi aux syndicats, à la protection de l'emploi, et, bien entendu, au salaire minimum proprement dit. Lorsque le salaire minimum est supérieur au salaire de plein emploi, l'emploi est rationné, et les jeunes supportent l'essentiel de ce rationnement ${ }^{5}$.

Finalement, les parents valorisent la situation économique de leurs enfants: le bien-être parental s'apprécie avec le revenu de l'enfant. En l'absence d'épargne (ou de legs), cette hypothèse revient à considérer la présence d'altruisme rationnel à la Barro (1974). Lorsque le revenu des enfants est un bien normal, toute aggravation de la situation économique de l'enfant se traduit par une intensification des transferts en sa direction. La normalité du bien "revenu de l'enfant" est assurée dans notre modèle par la prise en compte d'une fonction d'utilité Cobb-Douglas. L'intensité de l'altruisme est ainsi mesurée par un paramètre unique: le facteur d'altruisme, qui pondère l'utilité dérivée du revenu de l'enfant.

Notre principal mécanisme est le suivant. Au niveau microéconomique, l'offre de travail des vieux augmente avec le facteur d'altruisme et l'ampleur du sous-emploi subi par les jeunes ${ }^{6}$. Tout accroissement du facteur d'altruisme se traduit donc par une intensification des efforts productifs des parents. Comme le travail des jeunes et celui des vieux sont parfaitement substituables dans la production, la productivité marginale du travail de chacun diminue. La situation économique des jeunes s'en trouve détériorée: ou leur rémunération est plus faible - c'est le cas lorsque le salaire minimum est inférieur à la productivité marginale du travail -, ou le chômage s'aggrave - lorsque le salaire minimum sature. La baisse consécutive du revenu des jeunes intensifie encore les efforts des plus âgés, ce qui renforce le mécanisme initial.

Notre analyse implique que l'effort productif des vieux et le chômage des jeunes ten-

\footnotetext{
${ }^{3} \mathrm{Hu}(1979)$ et Michel et Pestieau (2000) analysent dans un cadre similaire (mais sans chômage) la décision de cessation d'activité. L'intensité du travail des vieux représente alors leur offre de travail.

${ }^{4}$ L'endogénéisation de l'intensité du travail des jeunes conduirait à la mise en exergue de nouveaux effets, sans altérer toutefois ceux que nous suggérons dans cette étude. Voire, par exemple, Thibault (2002).

${ }^{5}$ D'un point de vue empirique, le salaire minimum stricto sensu a des effets controversés sur l'emploi total, mais réduit indubitablement l'emploi des jeunes (voir Dolado et al, 1996, Bazen et Skourias, 1999, et Abowd et al, 2001).

${ }^{6}$ L'économie admet en fait deux régimes: un régime où les transferts descendants sont positifs et où l'offre de travail des vieux diminue avec le revenu des jeunes; un régime où la contrainte de transfert positif sature et où l'offre de travail des vieux est indépendante du revenu des jeunes.
} 
dent à se renforcer mutuellement. Ce type de complémentarité est caractéristique des mécanismes aux sources de fluctuations endogènes. Pour tirer parti de cette intuition, nous supposons dans une première extension que la productivité des travailleurs âgés est négativement reliée à leur taux de chômage passé - une illustration du phénomène plus général d'apprentissage. Nous mettons alors en exergue des cycles stationnaires de période 2 , où une situation marquée par un chômage des jeunes élevé, un effort productif important des travailleurs âgés, et des transferts inter-vivos massifs alterne avec la situation polaire où le chômage des jeunes, l'effort productif des vieux et les transferts inter-vivos sont plus réduits.

Nous proposons au cours d'une seconde extension l'endogénéisation du salaire minimum. Cette extension s'inscrit dans la lignée des travaux fondateurs de Saint-Paul voire son livre de 2001 pour une synthèse -, afférant à l'économie politique des institutions du marché du travail ${ }^{7}$. Nous supposons ainsi que la productivité d'un travailleur jeune est aléatoire, et que le niveau de la productivité n'affecte pas les chances individuelles d'emploi. Le salaire minimum joue alors le rôle d'une assurance sociale contre le risque d'être faiblement productif ${ }^{8}$. Nous donnons des conditions paramétriques pour lesquelles les salaires minima préférés des vieux comme des jeunes sont supérieurs à la productivité marginale du travail de plein emploi. Nous montrons également que le salaire minimum préféré par les jeunes n'est jamais inférieur au salaire minimum préféré des vieux. Le parent compense en effet par des transferts monétaires la baisse de revenu anticipé de l'enfant consécutive à l'augmentation du chômage. Le coût induit du salaire minimum est donc moindre pour l'enfant, et plus élevé pour le parent. Dans ce cadre, une augmentation du facteur d'altruisme se traduit généralement par une hausse du salaire minimum, un effort productif accru des parents, et une augmentation du chômage subi par les enfants.

Les idées développées dans le présent article renvoient à la littérature consacrée aux paradoxes de l'altruisme, littérature dont on pourra trouver une synthèse dans l'article de Cahuc et Kempf (2000). Le paradoxe le plus célèbre est celui connu sous l'appellation de dilemme du samaritain (cf. Buchanan (1975)). Il part de l'idée que l'amour du prochain entraîne des effets de risque moral, qui sont sources d'inefficacité. Dans le contexte de la redistribution et de l'assurance, Coate (1995) montre ainsi que l'incapacité des "riches" altruistes à s'engager à ne pas effectuer de dons envers les "pauvres" qui ne se sont pas assurés crée les conditions propices à une provision insuffisante d'assurance, et, en définitive, à une diminution du bien-être des pauvres. Notre analyse ressortit davantage

\footnotetext{
${ }^{7}$ On pourra également consulter Decreuse et Wigniolle (2002) dans le cas plus particulier des conflits d'intérêt intergénérationnels quant au niveau approprié du salaire minimum.

${ }^{8}$ Boadway et Marceau (1994) sont les premiers à avoir analysé le salaire minimum comme instrument efficace de redistribution. Agell (1999) insiste sur cette notion d'assurance sociale que l'on peut associer aux institutions du marché du travail.
} 
d'un autre paradoxe, qui a été étudié principalement par Stark (1989), et que Cahuc et Kempf (2000) qualifient de "paradoxe de l'altruisme local". Il correspond à l'idée qu'un altruisme qui ne s'exerce qu'au sein d'une communauté, peut engendrer des effets négatifs au niveau global. Cela provient de ce que la coopération au sein du groupe produit une externalité négative pour le reste de l"économie. Dans notre analyse, l'altruisme familial engendre ainsi une augmentation de l'offre de travail au sein de chaque famille, qui conduit à l'accroissement du chômage des jeunes.

La discussion s'organise comme suit. La section suivante présente un modèle à générations imbriquées où les individus âgés ont des préférences altruistes vis-à-vis de leur descendant et choisissent l'intensité de leur offre de travail en présence d'un salaire minimum. L'équilibre est analysé en section 3, où l'on met l'accent sur les liens entre facteur d'altruisme, comportement d'offre de travail et émergence du chômage. Les conséquences d'une relation négative entre la durée passée au chômage et la productivité des travailleurs âgés font l'objet de la section 4. Enfin, la détermination endogène ("politique") du salaire fait l'objet de la section 5 .

\section{Le modèle}

Nous décrivons un modèle à générations imbriquées sans capital physique composé de deux types d'individus : des propriétaires terriens qui vivent de leurs rentes et se transmettent de génération en génération les droits de propriété sur la terre, et des travailleurs qui ne possèdent pas de terres et se contentent du revenu de leur travail. Chacun de ces individus vit deux périodes et met au monde un enfant. Deux générations de travailleurs de taille identique et normalisée à un coexistent ainsi sur le marché du travail à chaque période $t$.

Chaque individu est doté à chaque période d'une unité de temps. Les décisions prennent place dans un environnement marqué par le sous-emploi. On suppose le mode de répartition du chômage suivant:

- au niveau intergénérationnel, les jeunes supportent l'intégralité de la charge du chômage (du moins tant que la quantité de jeunes le permet). Le marché du travail obéit ainsi au schéma insider/outsider usuel, à ceci près que les insiders coincident avec les travailleurs âgés, les outsiders étant naturellement composés des travailleurs jeunes.

- au niveau intragénérationnel, le chômage est uniformément réparti sur l'ensemble des travailleurs jeunes. La durée de l'épisode de chômage subi par chaque individu est donc déterministe et égale au taux de chômage de l'économie. Cette hypothèse paraît relativement réaliste compte tenu de la longueur implicite d'une période de vie, et de la banalité du phénomène de chômage parmi les jeunes.

Une personne jeune obtient ainsi le revenu $y_{t} \equiv\left(1-u_{t}\right) w_{t}^{j}$, où $u_{t}$ est la durée de son 
épisode de chômage et $w_{t}^{j}$ est le salaire par unité de temps travaillée qu'il perçoit. D'autre part, cette personne reçoit un transfert financier $x_{t}$ de la part de son parent. Il n'y a pas de possibilité d'épargner, de sorte que le revenu du travail $y_{t}$ et le transfert financier $x_{t}$ sont intégralement affectés à la consommation :

$$
c_{t}=y_{t}+x_{t}
$$

Pendant une seconde période de vie, cette personne choisit la fraction de temps $l_{t+1}$ passé à travailler. En contrepartie de ce travail, elle obtient le revenu $l_{t+1} w_{t+1}^{v}$, où $w_{t+1}^{v}$ est le salaire des travailleurs âgés. Ce revenu lui sert à financer sa consommation $d_{t+1}$ ainsi que le transfert $x_{t+1}$ versé à son enfant :

$$
x_{t+1}+d_{t+1}=l_{t+1} w_{t+1}^{v}
$$

L'utilité du membre représentatif de la génération $t$ dépend de ses consommations de première et deuxième période de vie, et de son loisir $\ell_{t+1} \equiv 1-l_{t+1}$. En outre nous supposons que l'utilité dépend du niveau de revenu dont dispose l'enfant, c'est-à-dire la somme du revenu autonome $y_{t+1}$ et du transfert $x_{t+1}$. Dans notre contexte sans épargne, cette hypothèse revient à considérer une forme d'altruisme rationnel ${ }^{9}$ à la Barro (1974). Nous utiliserons une forme log-linéaire de l'utilité :

$$
U_{t}=\ln c_{t}+\alpha \ln d_{t+1}+\beta \ln \left(y_{t+1}+x_{t+1}\right)+\gamma \ln \ell_{t+1}
$$

où $\alpha$ est le facteur de préférence pour le futur, $\beta$ est le facteur d'altruisme, et $\gamma$ représente la préférence pour le loisir. Le membre représentatif de la génération $t$ maximise l'utilité (3) sous les contraintes de revenu (1) et (2), et la contrainte d'allocation du temps de vie de deuxième période entre travail loisir $0 \leq \ell_{t+1} \equiv 1-l_{t+1} \leq 1$. D'autre part, nous supposons que le parent n'a pas le contrôle des ressources de son enfant. Il ne peut donc lui extraire de revenu, de sorte qu'il doit également respecter la contrainte de transfert positif $x_{t+1} \geq 0$.

On désigne par $\widetilde{x}_{t+1}$ et $\widetilde{l}_{t+1}$ les solutions de ce programme.

Proposition 1 Soit $\Delta\left(u_{t+1}, w_{t+1}^{j} / w_{t+1}^{v}\right)=\beta-(\alpha+\gamma)\left(1-u_{t+1}\right) w_{t+1}^{j} / w_{t+1}^{v}$

(i) Si $\Delta_{t+1}<0$,

$$
\begin{aligned}
\widetilde{x}_{t+1} & =0 \\
\widetilde{l}_{t+1} & =l^{c} \equiv \alpha /(\alpha+\gamma)
\end{aligned}
$$

\footnotetext{
${ }^{9}$ En effet, l'enfant consomme la totalité de son revenu. Le parent ne peut donc altérer son comportement; il est conduit à maximiser (3), à une constante près. On peut maintenir l'objectif (3) en présence d'épargne, mais ce n'est plus de l'altruisme à la Barro puisque le parent ne tient pas compte de l'utilisation du revenu par l'enfant. Il s'agit alors d'une forme myope d'altruisme (voir Lambrecht, 2003, et Lambrecht et al, 2005).
} 
(ii) Si $\Delta_{t+1} \geq 0$,

$$
\begin{aligned}
& \widetilde{x}_{t+1}=x\left(w_{t+1}^{v}, w_{t+1}^{j}, u_{t+1}\right) \equiv \frac{w_{t+1}^{v}}{\alpha+\beta+\gamma} \Delta\left(u_{t+1}, w_{t+1}^{j} / w_{t+1}^{v}\right) \\
& \widetilde{l}_{t+1}=l^{n c}\left(w_{t+1}^{v} / w_{t+1}^{j}, u_{t+1}\right) \equiv l^{c}+\frac{\gamma}{\alpha+\gamma} \frac{x\left(w_{t+1}^{v}, w_{t+1}^{j}, u_{t+1}\right)}{w_{t+1}^{v}}
\end{aligned}
$$

L'offre de travail des vieux dépend de la contrainte de transfert positif. Celle-ci est inactive lorsque le facteur d'altruisme $\beta$, le taux de chômage des jeunes $u_{t+1}$ et le différentiel salarial $w_{t+1}^{v} / w_{t+1}^{j}$ sont suffisamment grands. Lorsque ces conditions ne sont pas remplies, le parent aimerait ponctionner son enfant, ce qui lui permettrait de travailler moins. Son offre de travail est ainsi contrainte et vaut $l^{c}$; elle est indépendante du revenu de l'enfant. Lorsque $\Delta_{t+1} \geq 0$, l'offre de travail vaut $l^{n c}\left(w_{t+1}^{v} / w_{t+1}^{j}, u_{t+1}\right)$. Elle augmente avec le différentiel salarial $\left(l_{1}^{n c}>0\right)$ et avec le taux de chômage $u_{t+1}\left(l_{2}^{n c}>0\right)$. Cette dernière propriété signifie que l'offre de travail des vieux s'accroît lorsque la situation économique de leur enfant se dégrade. Or, comme nous allons le montrer, l'offre de travail des vieux est un déterminant clé des difficultés rencontrées par les jeunes.

La production dépend des quantités de facteur non-reproductible $T$ et de travail des jeunes $L_{t}^{j}$ et des vieux $L_{t}^{v}$ selon

$$
Y_{t}=G\left(T, L_{t}^{j}, L_{t}^{v}\right)=G\left(T, L_{t}^{j}+a L_{t}^{v}\right)
$$

où $a>1$ est un facteur de productivité spécifique aux travailleurs âgés; il est lié aux effets d'apprentissage glanés par les vieux au cours de la période précédente ${ }^{10}$. Nous supposons ainsi que le travail est homogène dans la production. La firme choisit les quantités de chaque type de travail de façon à maximiser son profit. Ainsi,

$$
\begin{aligned}
w_{t}^{j} & =G_{2}\left(T ; L_{t}^{j}+a L_{t}^{v}\right) \\
w_{t}^{v} & =a w_{t}^{j}
\end{aligned}
$$

La firme ajuste l'emploi jusqu'à ce que la productivité marginale de chaque travail coincide avec le salaire versé. Ce comportement implique que le salaire relatif des travailleurs âgés est proportionnel à celui des jeunes, quel que soit le mode de détermination des salaires. Si le salaire dépasse la productivité marginale du travail de plein emploi, il y a émergence de chômage. Compte tenu de nos hypothèses précédentes, ce chômage affecte prioritairement les travailleurs jeunes. De manière à alléger les notations, nous ne ferons plus apparaitre la dépendance qui lie la production au facteur terre. Nous posons ainsi $F\left(L_{t}^{j}+a L_{t}^{v}\right) \equiv G\left(T ; L_{t}^{j}+a L_{t}^{v}\right)$.

\footnotetext{
${ }^{10} \mathrm{Ce}$ processus est explicitement modélisé en section 4 .
} 


\section{Altruisme, travail des vieux et chômage des jeunes}

Dans cette section, nous étudions les différents régimes de l'économie. Le principal résultat est qu'une hausse du facteur d'altruisme augmente l'offre de travail des vieux, ce qui déprécie la position économique des jeunes, au travers d'une baisse de salaire ou d'une hausse du chômage.

\subsection{Equilibre}

On désigne par $w_{\min }, w^{*}$ et $\widehat{w}$ (resp.) le salaire minimum, le salaire effectif, et le salaire notionnel qui solde le marché du travail. Ce dernier est défini par l'égalité suivante :

$$
\widehat{w}=F^{\prime}(1+a \widetilde{l}(a, 0))
$$

Le salaire notionnel est une fonction décroissante de l'offre de travail des individus âgés. Il est ainsi commode de distinguer le salaire notionnel selon que $\widetilde{l}$ est contrainte ou non. Soit:

$$
\begin{aligned}
\widehat{w}^{c} & =F^{\prime}\left(1+a l^{c}\right)=F^{\prime}\left(1+\frac{\alpha}{\alpha+\gamma} a\right) \\
\widehat{w}^{n c} & =F^{\prime}\left(1+a l^{n c}\right)=F^{\prime}\left(\frac{\alpha+\beta}{\alpha+\beta+\gamma}(1+a)\right)
\end{aligned}
$$

Comme $l^{n c}>l^{c}$ tant que $x>0$, on a $\widehat{w}^{c}>\widehat{w}^{n c}$. Lorsque l'offre de travail n'est pas contrainte, le salaire notionnel décroît avec $a$ et $\beta$. Deux cas de figure sont susceptibles d'émerger:

1. Si $w_{\min } \leq \widehat{w}, w^{*}=\widehat{w}$ et $u^{*}=0$. C'est l'équilibre de plein emploi.

2. Si $w_{\min }>\widehat{w}, w^{*}=w_{\min }$, on a un équilibre avec chômage et le taux de chômage est défini implicitement par:

$$
w_{\min }=F^{\prime}\left(1-u^{*}+a \widetilde{l}\left(a, u^{*}\right)\right)
$$

Le taux de chômage délivré par cette expression diffère selon la nature de l'offre de travail. Avec des notations évidentes, on a:

$$
\begin{aligned}
u_{c} & =1+\frac{\alpha}{\alpha+\gamma} a-F^{\prime-1}\left(w_{\min }\right) \\
u_{n c} & =1+a-\frac{\alpha+\beta+\gamma}{\alpha+\beta} F^{\prime-1}\left(w_{\min }\right)
\end{aligned}
$$

Proposition 2 Soit $\bar{w}=F^{\prime}\left(\frac{\alpha+\beta}{\alpha+\gamma} a\right)$ et $w_{\max }=F^{\prime}\left(\frac{\alpha+\beta}{\alpha+\beta+\gamma} a\right)$

(i) Si $\beta a \geq \alpha+\gamma, u^{*}=\left\{\begin{array}{l}0 \text { si } w_{\min } \leq \widehat{w}^{n c} \\ u_{n c} \text { sinon }\end{array}\right.$ 
(ii) Si $\beta a<\alpha+\gamma, u^{*}=\left\{\begin{array}{l}0 \text { si } w_{\min } \leq \widehat{w}^{c} \\ \left.\left.u_{c} \text { si } w_{\min } \in\right] \widehat{w}^{c}, \bar{w}\right] \\ u_{n c} \text { sinon }\end{array}\right.$

Lorsque $\beta a \geq \alpha+\gamma, \Delta \geq 0$ et la contrainte de transfert positif ne sature jamais. Tant que $w_{\min }$ reste inférieur à $\widehat{w}^{n c}$, il n'y a pas de chômage. Puis le taux de chômage $u_{n c}$ augmente pour atteindre 1 lorsque $w_{\min }=w_{\max }$. En revanche, lorsque $\beta a<\alpha+\gamma$, la contrainte de transfert positif sature jusqu'à ce que le taux de chômage des jeunes atteigne une valeur suffisamment élevée, définie par $w_{\min }=\bar{w}$. La figure suivante illustre ces résultats:

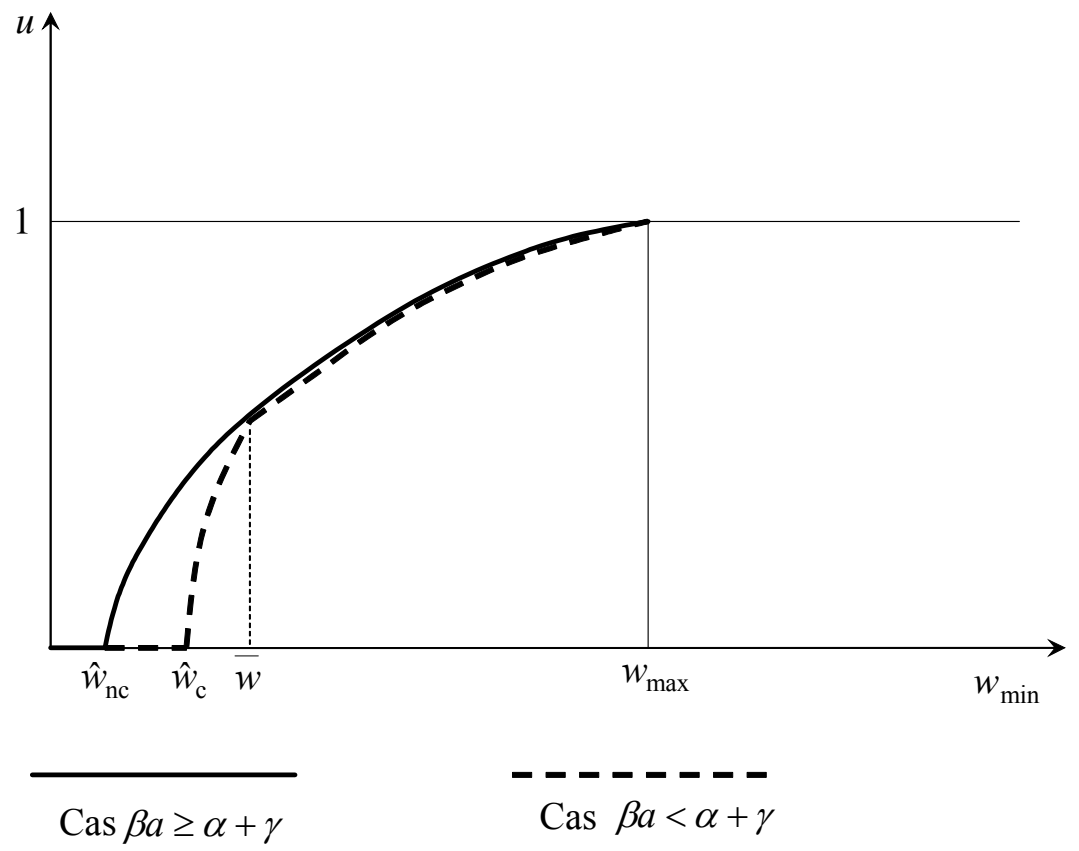

Figure 2 : le chômage en fonction du salaire minimum

\subsection{Le rôle pervers de l'altruisme}

Nous analysons maintenant les effets macroéconomiques du facteur d'altruisme. A cette fin, on note $u_{n c}=u_{n c}(\beta)$ et $l^{n c}=l^{n c}(\beta, u)$ pour souligner la dépendance vis-à-vis du paramètre $\beta$.

Proposition 3 Soit $\beta_{1}=\frac{\alpha+\gamma}{a}, \beta_{2}=\frac{(\alpha+\gamma) F^{\prime-1}\left(w_{\min }\right)-\alpha(1+a)}{1+a-F^{\prime-1}\left(w_{\min }\right)}$ et $\beta_{3}=\frac{\alpha+\gamma}{a} F^{\prime-1}\left(w_{\min }\right)-\alpha$.

(i) Si $w_{\min }<F^{\prime}(1+a)$,

$$
u^{*}=0, \text { et } l^{*}=\left\{\begin{array}{l}
l^{c} \text { si } \beta<\beta_{1} \\
l^{n c}(\beta, 0) \text { sinon }
\end{array}\right.
$$


(ii) Si $w_{\min } \in\left[F^{\prime}(1+a), F^{\prime}\left(1+\frac{\alpha}{\alpha+\gamma} a\right)[\right.$,

$$
u^{*}=\left\{\begin{array}{l}
0 \text { si } \beta<\beta_{2} \\
u_{n c}(\beta) \text { sinon }
\end{array} \quad \text { et } l^{*}=\left\{\begin{array}{l}
l^{c} \text { si } \beta<\beta_{1} \\
l^{n c}(\beta, 0) \text { si } \beta \in\left[\beta_{1}, \beta_{2}[\right. \\
l^{n c}\left(\beta, u_{n c}(\beta)\right) \text { si } \beta \geq \beta_{2}
\end{array}\right.\right.
$$

(iii) Si $w_{\min } \in\left[F^{\prime}\left(1+\frac{\alpha}{\alpha+\gamma} a\right), F^{\prime}\left(\frac{\alpha}{\alpha+\gamma} a\right)\right]$,

$$
u^{*}=\left\{\begin{array}{l}
u_{c} \text { si } \beta<\beta_{3} \\
u_{n c}(\beta) \text { sinon }
\end{array} \text { et } l^{*}=\left\{\begin{array}{l}
l^{c} \text { si } \beta<\beta_{3} \\
l^{n c}\left(\beta, u_{n c}(\beta)\right) \text { si } \beta \geq \beta_{3}
\end{array}\right.\right.
$$

Les figures $3 \mathrm{a}$, 3b et $3 \mathrm{c}$ illustrent les différents résultats obtenus dans la proposition 3.

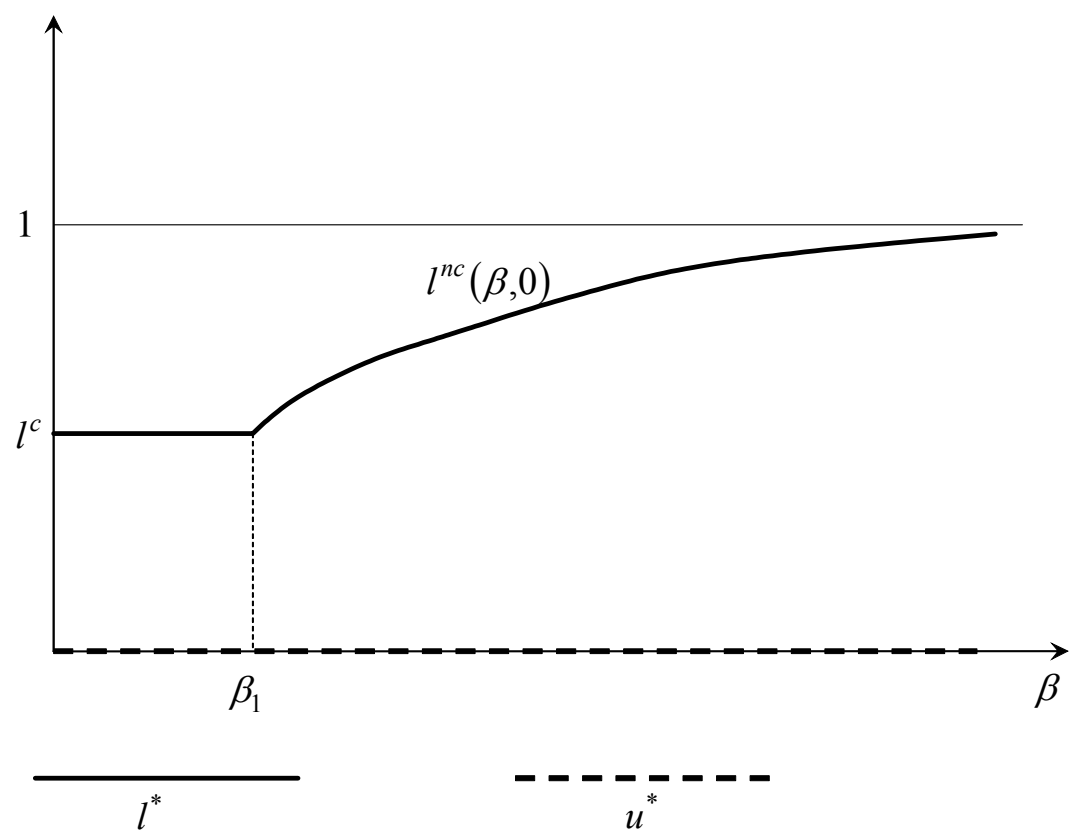

Figure 3a: le cas $w_{\min }<F^{\prime}(1+a)$ 


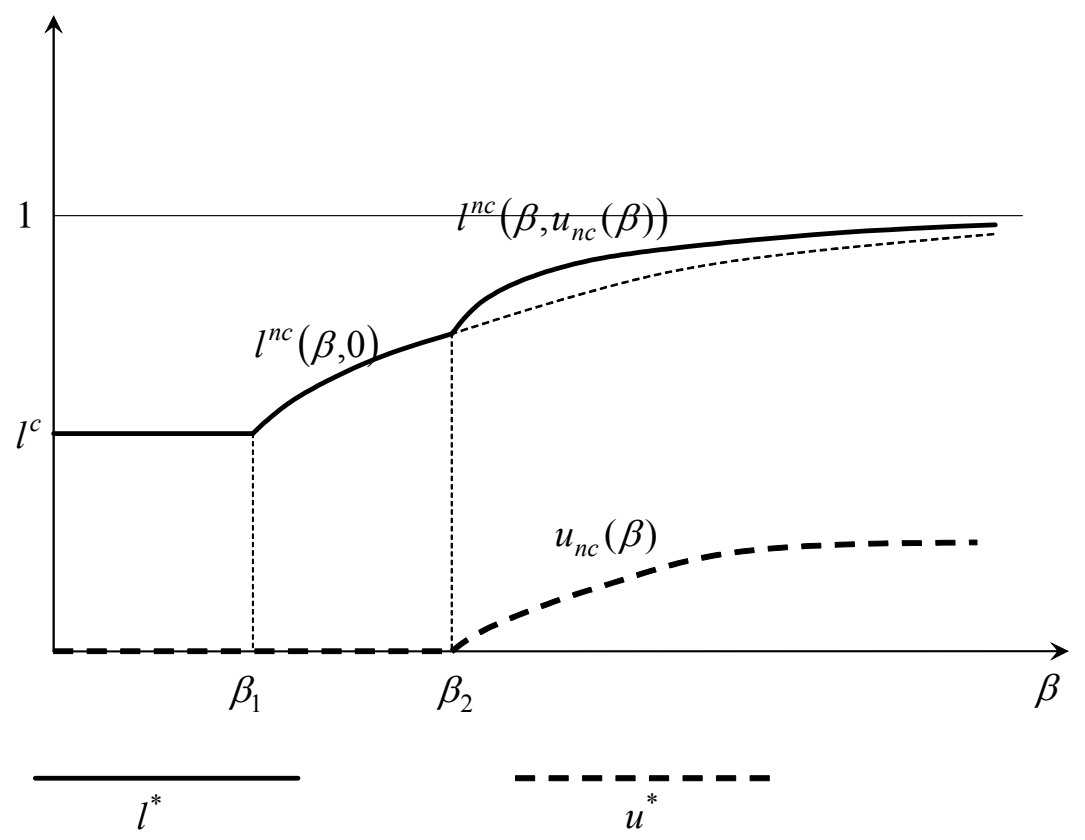

Figure $3 \mathrm{~b}$ : le cas $w_{\min } \in\left[F^{\prime}(1+a), F^{\prime}\left(1+\frac{\alpha}{\alpha+\gamma} a\right)[\right.$

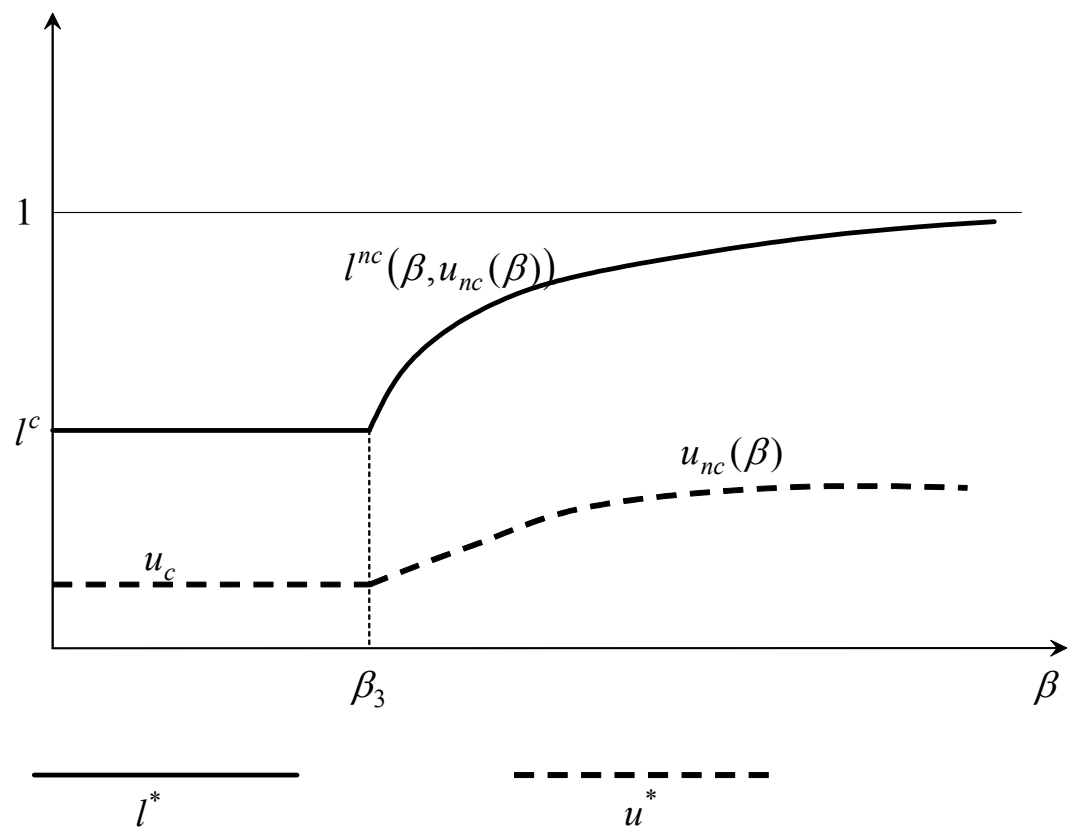

Figure $3 \mathrm{c}:$ le cas $w_{\min } \in\left[F^{\prime}\left(1+\frac{\alpha}{\alpha+\gamma} a\right), F^{\prime}\left(\frac{\alpha}{\alpha+\gamma} a\right)\right]$

Le facteur d'altruisme ne joue un rôle qu'au travers de l'offre de travail des individus âgés. Or, celle-ci tend à augmenter avec $\beta$, ce qui a pour effet de réduire le taux de salaire tant que celui-ci dépasse le salaire minimum, et d'accroître le taux de chômage 
lorsque ce n'est plus le cas. Ainsi, dans le but d'améliorer la situation des économique des travailleurs jeunes, les travailleurs âgés sont responsables de la baisse de leur salaire, ou de la hausse de leur chômage. La proposition 2 ne fait que préciser cette intuition, en distinguant les plages de paramètres pour lesquels la contrainte de transfert positif sature et pour lesquels il y a plein emploi. Cette véritable schizophrénie des comportements des vieux vis-à-vis des jeunes donne son titre à ce travail.

Deux hypothèses jouent un rôle important dans la genèse de ce résultat: elles affèrent aux fonctionnements du marché du crédit et du marché du travail.

Nous avons exclu d'emblée la possibilité d'une épargne, tout aussi bien négative que positive. Si les jeunes avaient accès au marché du crédit, ils pourraient être tentés de transférer des ressources dans le futur, substituant ainsi l'épargne au travail. Ce type de transfert serait d'autant plus important que les ressources disponibles en première période de vie seraient abondantes. Or, l'ampleur de ces ressources est directement reliée au facteur d'altruisme parental. Ainsi, l'effet direct de l'altruisme parental qui pousse les parents à intensifier leur effort serait atténué par un effet d'équilibre général inverse induit par l'augmentation du revenu des parents ${ }^{11}$.

Nous avons postulé que les travailleurs âgés bénéficie d'une priorité à l'embauche/à la conservation de l'emploi. D'une certaine manière, cette hypothèse n'est pas essentielle au fonctionnement du modèle. Si le partage générationnel du chômage était plus équitable, une augmentation de l'offre de travail des vieux se traduirait par l'aggravation simultanée du chômage des jeunes et de celui de leurs parents. Ainsi, l'intensité de l'amour filial conditionnerait la distribution complète des taux de chômage par groupe d'âge, et pas seulement le chômage des jeunes. Il n'empêche que le principal message perdurerait: toute augmentation du facteur d'altruisme a des conséquences perverses sur la situation économique de ceux que l'on désire aider. En revanche, l'article perdrait son ton polémique, puisqu'il ne serait plus capable de rendre compte de la situation spécifique des jeunes au regard du chômage. Une manière simple de justifier la protection de l'emploi dont bénéficie les vieux au sein du modèle consiste à supposer que celle-ci se décide avant tout au sein de chaque firme, c'est-à-dire sans possibilité d'internalisation des effets pervers sur les plus jeunes. L'employeur choisit l'emploi (les nombres de travailleurs embauchés et licenciés), à charge pour un syndicat composé de travailleurs représentatifs de déterminer qui est licencié, et qui est embauché. Les travailleurs plus âgés étant les plus nombreux, leurs intérêts sont privilégiés par le syndicat, ce qui explique leur faible exposition au risque chômage.

\footnotetext{
${ }^{11}$ L'argument renvoie à Thibault (2004), qui examine un modèle avec offre de travail endogène des jeunes, legs, et non travail des vieux. L'offre de travail augmente avec le legs que l'on entend laisser, mais diminue avec le legs que l'on perçoit. A l'équilibre stationnaire, les deux legs coïncident, ce qui explique l'ambiguïté des liens entre legs et offre de travail stationnaires.
} 


\section{Apprentissage par la pratique et dynamique du chômage}

L'offre de travail des individus âgés dépend de la préférence pour le loisir, du salaire minimum et du paramètre d'altruisme. Mais elle est également une fonction croissante du paramètre $a$ de productivité spécifique au travailleur âgé. Ce paramètre renvoie aux effets d'apprentissage dont ont bénéficié les travailleurs plus expérimentés. De ce point de vue, il devrait être a priori d'autant plus faible que le taux de chômage subi étant jeune était élevé. Nous analysons les conséquences de cet argument.

Nous supposons ainsi que

$$
a \equiv a\left(u_{t-1}\right), a^{\prime}<0 \text { et } a(1)>1
$$

Le salaire notionnel dépend à chaque période du taux de chômage qui prévalait la période précédente selon:

$$
\widehat{w}_{t}=F^{\prime}\left(1+a\left(u_{t-1}\right) \widetilde{l}\left(a\left(u_{t-1}\right), 0\right)\right)
$$

Nous limiterons l'analyse aux cas où l'altruisme est opérant. A cette fin, on suppose que $\beta a(1) \geq \alpha+\gamma$. Le salaire notionnel vaut ainsi

$$
\widehat{w}_{t}=F^{\prime}\left(\frac{\alpha+\beta}{\alpha+\beta+\gamma}\left(1+a\left(u_{t-1}\right)\right)\right)=\widehat{w}\left(u_{t-1}\right)
$$

Il s'agit d'une fonction strictement croissante du taux de chômage de la période passée (voir figure 2). Cette dépendance reflète d'une part l'effet direct de la productivité des travailleurs âgés, mais aussi son impact sur l'offre de travail des vieux. 


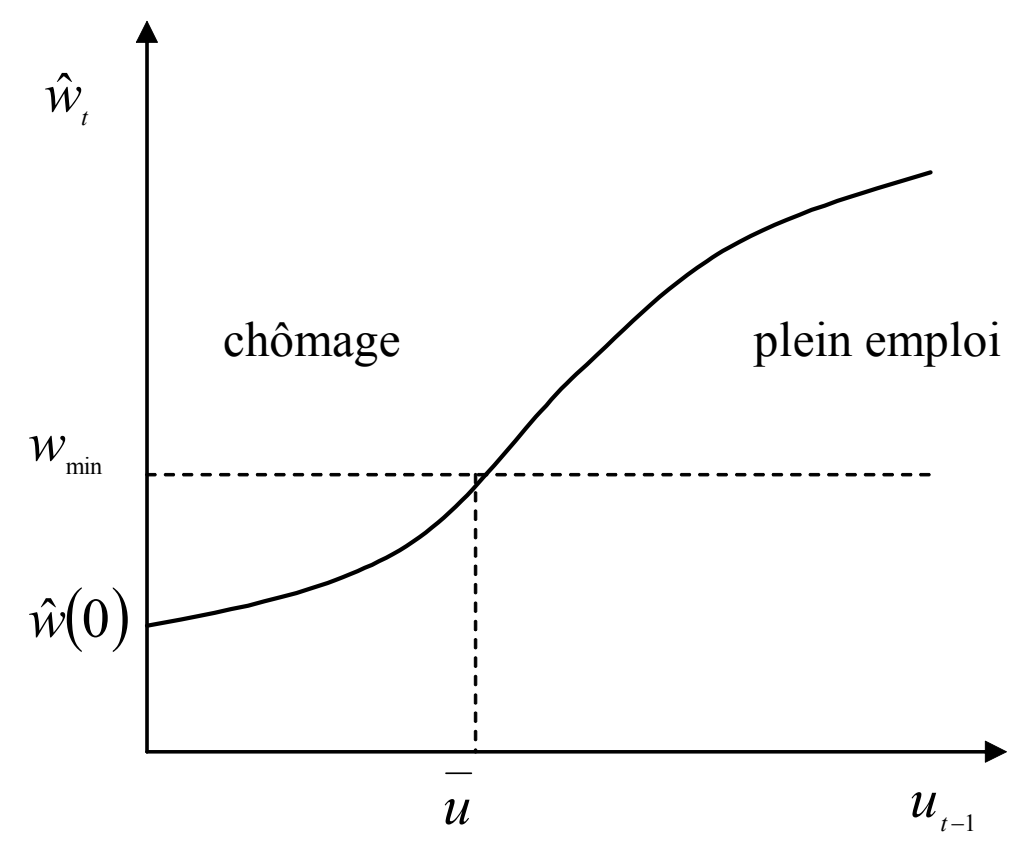

Fig. 4 : Salaire notionnel et chômage

Comme précédemment, la situation du marché du travail dépend de la valeur du salaire minimum. Si $w_{\min } \leq \widehat{w}_{t}$, le salaire effectif $w_{t}$ est égal au salaire notionnel $\widehat{w}_{t}$ et le taux de chômage $u_{t}=0$. En revanche, lorsque $w_{\min }>\widehat{w}_{t}$, l'équilibre du marché du travail réclame un taux de chômage strictement positif. Nous ne nous intéresserons qu'aux situations où le salaire minimum peut devenir contraignant, soit $w_{\min } \geq \widehat{w}(0)$. Dans ce cas, il existe un unique taux de chômage passé $\bar{u}$ au-delà duquel l'équilibre de la période courante est l'équilibre de plein emploi, et en-deça duquel l'équilibre est caractérisé par un taux de chômage non nul. Formellement, ce taux de chômage résout $\widehat{w}(\bar{u})=w_{\min }$. Soit,

$$
\bar{u}=a^{-1}\left\{\frac{\alpha+\beta+\gamma}{\alpha+\beta} F^{\prime-1}\left(w_{\min }\right)-1\right\}
$$

Lorsque le salaire minimum dépasse le salaire notionnel, le taux de chômage résout:

$$
w_{\min }=F^{\prime}\left(1-u_{t}+a\left(u_{t-1}\right) \widetilde{l}\left(a\left(u_{t-1}\right), u_{t}\right)\right)
$$

Cette relation détermine le taux de chômage courant comme une fonction strictement décroissante du taux de chômage de la période précédente, i.e. $u_{t}=\max \left\{\min \left\langle\psi\left(u_{t-1}\right), 1\right\rangle, 0\right\}$, où

$$
\psi(u)=1+a(u)-\frac{\alpha+\beta+\gamma}{\alpha+\beta} F^{\prime-1}\left(w_{\min }\right)
$$

Proposition 4 Il existe un unique état stationnaire. Ou $u_{t}$ converge vers $u^{*}$ par oscillations, ou $u_{t}$ converge vers un cycle de période 2. 
L'économie peut converger vers son unique état stationnaire ; mais il est également possible qu'elle converge vers un cycle stationnaire de période 2. Deux types de cycles peuvent être distingués. Les cycles intérieurs font alterner l'économie entre deux niveaux de chômage strictement positifs. L'occurence de ces cycles ne dépend que des propriétés de la fonction $\psi$ sur l'intervalle ouvert $] 0, \bar{u}[$. Les cycles frontières font alterner l'économie entre une situation de plein emploi et une situation avec chômage strictement positif. De tels cycles existent dès lors que $\psi(0)>\bar{u} .^{12}$

On trouve le même mécanisme en amont de ces deux types de cycles. Prenons le cas d'un cycle frontière. L'économie alterne alors entre deux situations très contrastées. La première avec un taux de chômage élevé et une offre de travail des vieux importante; la seconde avec le plein emploi et une offre de travail des vieux plutôt réduite. Considérons une période $t$ quelconque, et supposons que l'offre de travail des vieux à cette période permet le plein emploi des jeunes. Ceux-ci accumulent un maximum de capital humain, qui leur permet de bénéficier d'une productivité maximale lors de leur seconde période de vie. Afin de bénéficier de cette productivité élevée, et pour en faire profiter leur enfant, ils offrent abondamment leur travail en période $t+1$. Le salaire notionnel passe sous le salaire minimum, et les jeunes connaissent le chômage. A la période $t+2$, le différentiel de productivité entre jeunes et vieux n'est pas aussi élevé qu'il l'était à la période précédente. Le prix implicite du loisir est donc plus élevé, ce qui pousse les vieux à choisir une faible offre de travail. Ce comportement est compatible avec le plein emploi des jeunes, et le cycle continue.

\section{$5 \quad$ D'où vient le salaire minimum?}

Les résultats exposés jusqu'ici reposent sur l'existence d'un salaire minimum éventuellement contraignant. Cette hypothèse est bien entendu réaliste, mais il nous semble que nos arguments prendraient davantage de vigueur si ils étaient compatibles avec une détermination endogène du salaire minimum. Nous étudions dans cette section le soutien politique que peut trouver le salaire minimum. Nous montrons que ce soutien est probablement plus élevé parmi les jeunes que parmi les vieux. Ce résultat s'obtient au prix d'une hypothèse supplémentaire, concernant l'incertitude sur les revenus d'un travailleur jeune.

On suppose que les travailleurs jeunes ont une productivité aléatoire, ce que l'on résume de la façon suivante: avec probabilité $p$, le travailleur est de type 1 "compétent", alors qu'il est de type 0 "incompétent" avec probabilité $1-p$. Soit $b^{i}$ l'efficacité du travail

\footnotetext{
${ }^{12}$ Un tel cas survient lorsque $a$ est concave et $a^{\prime}(0)<-1$.
} 
de l'enfant de type $i$ :

$$
b^{i}=\left\{\begin{array}{l}
b<1 \text { si } i=0 \\
1 \text { si } i=1
\end{array}\right.
$$

On suppose par ailleurs qu'il n'existe pas de système d'assurance permettant d'assurer le risque d'avoir un enfant incompétent ${ }^{13}$. Pour simplifier, les travailleurs âgés ont tous la même productivité ${ }^{14}$.

Le calendrier est le suivant. En début de période, les individus votent sur le niveau du salaire minimum. Puis le type se révèle, et chaque individu participe à la production selon sa productivité et son statut. Enfin, les parents choisissent l'ampleur des transferts en direction de leur enfant.

La production vaut maintenant:

$$
Y=G\left(T, b L_{0}^{j}+L_{1}^{j}+a L^{v}\right)
$$

On pose à nouveau $F\left(b L_{0}^{j}+L_{1}^{j}+a L^{v}\right) \equiv G\left(T, b L_{0}^{j}+L_{1}^{j}+a L^{v}\right)$. Nous adopterons par la suite une spécification Cobb-Douglas ${ }^{15}$, de sorte que

$$
G\left(T, b L_{0}^{j}+L_{1}^{j}+a L^{v}\right)=T^{1-\sigma}\left(b L_{0}^{j}+L_{1}^{j}+a L^{v}\right)^{\sigma}
$$

avec $0<\sigma<1$. Les rémunérations de chaque type de travailleur dépendent de la productivité marginale du travail selon:

$$
w^{v}=\frac{a}{b} w_{0}^{j}=a w_{1}^{j}
$$

A l'instar des sections précédentes, le mot "salaire" désigne la rémunération du travailleur le moins productif, soit $w \equiv w_{0}^{j}$, de sorte que $w^{v}=\frac{a}{b} w$ et $w_{i}^{j}=\frac{b^{i}}{b} w, i=0,1$.

Le comportement des parents devient contingent au type de l'enfant. On se limite volontairement au cas où l'altruisme est toujours opérant ${ }^{16}$, ce qui revient à imposer qu'il l'est dans le cas où l'enfant de type compétent connaît le plein emploi, i.e. $\beta a \geq(\alpha+\gamma)$.

\footnotetext{
${ }^{13}$ Rien n'interdit l'existence d'un tel système dans le modèle. Nous nous réfugions donc derrière des arguments de réalisme, postulant l'existence de phénomènes d'aléa moral non modélisés.

${ }^{14}$ Cette hypothèse ne joue aucun rôle majeur; elle sert uniquement à réduire le nombre de combinaisons possibles productivité du parent/productivité de l'enfant.

${ }^{15}$ Nous conserverons toutefois aussi souvent que possible la notation $F$ pour économiser de l'espace.

${ }^{16}$ Lorsque l'altruisme n'est pas opérant, les parents désirent la mise en place d'un salaire minimum élevé qui leur permet d'obtenir au niveau agrégé ce qu'ils ne parviennent pas à obtenir au sein de leur famille. Ce cas ne nous intéresse guère puisqu'il entre en contradiction avec le thème majeur de ce travail, à savoir que les mauvaises conditions rencontrées par les jeunes sur le marché du travail sont une conséquence malheureuse du trop plein d'altruisme des parents.
} 
On obtient ainsi:

$$
\begin{aligned}
x^{i}(u) & =\frac{a}{b} \frac{w}{\alpha+\beta+\gamma}\left[\beta-(\alpha+\gamma)(1-u) \frac{b^{i}}{a}\right] \\
l^{i}(u) & =\frac{1}{\alpha+\beta+\gamma}\left[\alpha+\beta-\gamma(1-u) \frac{b^{i}}{a}\right] \\
d^{i}(u) & =\frac{\alpha}{\alpha+\beta+\gamma} \frac{a}{b} w\left[1+(1-u) \frac{b^{i}}{a}\right]
\end{aligned}
$$

Ces expressions impliquent que le transfert en direction de l'enfant et l'offre de travail sont des fonctions décroissantes de la productivité de l'enfant, alors que la consommation de deuxième période en est une fonction croissante. L'utilité indirecte ex-post (c'est-à-dire une fois le type de l'enfant révélé) obtenue par un parent dont l'enfant est de type $i$ vaut donc:

$$
v^{i}=(\alpha+\beta) \ln w+(\alpha+\beta+\gamma) \ln \left(a+(1-u) b^{i}\right)+c t e
$$

Alors que celle de son enfant est:

$$
z^{i}=\ln w+\ln \left(a+(1-u) b^{i}\right)+c t e
$$

Le bien-être des parents comme celui des enfants augmente avec l'efficacité productive des enfants. L'obtention d'un enfant de mauvais type est donc synonyme de perte de bien-être. En présence d'aversion pour le risque, ce phénomène induit une demande d'assurance de la part des deux groupes de travailleurs.

Le salaire notionnel est défini par:

$$
\widehat{w}=b F^{\prime}\left(b p+1-p+a\left(p l^{0}(0)+(1-p) l^{1}(0)\right)\right)
$$

Compte tenu de (31),

$$
\widehat{w}=b F^{\prime}\left(\frac{\alpha+\beta}{\alpha+\beta+\gamma}(a+p b+1-p)\right)
$$

Pour un niveau de salaire minimum donné supérieur au salaire notionnel, le taux de chômage des jeunes obéit à

$$
w_{\min }=b F^{\prime}\left(b p(1-u)+(1-p)(1-u)+a\left(p l^{0}(u)+(1-p) l^{1}(u)\right)\right)
$$

Compte tenu de (31),

$$
w_{\min }=b F^{\prime}\left(\frac{\alpha+\beta}{\alpha+\beta+\gamma}(a+(p b+1-p)(1-u))\right)
$$

Il y a trois groupes d'individus: les travailleurs jeunes, les travailleurs âgés, et les propriétaires terriens. Chacun d'entre eux formule un voeu quant au niveau du salaire minimum 
qui prévaut lors de la période courante. Ce voeu résulte bien entendu de la maximisation de l'espérance d'utilité, étant entendu qu'il est parfaitement équivalent de choisir le niveau du salaire minimum ou le taux de chômage des jeunes. On note $\widehat{u}^{k}$ le taux de chômage des jeunes préféré par les individus du groupe $k=j, v, r-r$ pour "rentier".

\section{Proposition 5 (i) $\widehat{u}^{r}=0$}

(ii) $\widehat{u}^{j} \geq \widehat{u}^{v}$

(iii) $\widehat{u}^{j}>\widehat{u}^{v}>0$ lorsque $\sigma$ et $\gamma$ sont suffisamment petits

Les propriétaires terriens inclinent au plein emploi. Le plein emploi maximise en effet la productivité marginale du facteur terre. Mais le principal résultat, c'est qu'à la fois travailleurs jeunes et travailleurs âgés peuvent désirer un taux de chômage des jeunes strictement positif. La raison tient au rôle assuranciel du salaire minimum : un enfant de type incompétent induit une perte de bien-être à la fois pour l'enfant et pour son parent. La mise en place d'un salaire minimum supérieur au salaire de plein emploi maintient le revenu familial dans cette éventualité. Remarquons que l'argument ne fonctionne que dans la mesure où enfants de mauvais et de bon types partagent le chômage induit par le salaire minimum. Si les enfants de bon type étaient exemptés du chômage (parce qu'ils sont plus productifs), une hausse du salaire minimum se traduirait par une baisse du revenu familial, et il n'existerait pas de soutien politique pour une telle mesure.

La proposition 5 met en avant un autre résultat important: le salaire minimum préféré des travailleurs âgés est inférieur à celui des travailleurs jeunes. Les jeunes anticipent en effet que le chômage induit par un salaire minimum élevé sera compensé par des transferts importants de la part de leur parent; ce qui les pousse à accepter de subir un taux de chômage plus élevé. Du point de vue des parents, le mécanisme est le même, à ceci près que le transfert s'accompagne nécessairement d'une réduction de leur temps de loisir et de leur consommation. C'est pourquoi ils inclinent à voter pour un salaire minimum plus faible. Un corollaire de ce résultat est que le groupe des travailleurs âgés fait office d'agent médian dans cette analyse. De ce point de vue, le salaire minimum effectif constaté dans les économies réelles refléterait les préférences de ce groupe de travailleurs. Cette idée est assez répandue dans la littérature (voire en particulier Sobel, 1999, pour une application empirique) ; il est toutefois important de signaler qu'elle dérive ici d'un principe profondément différent du principe habituel.

A notre connaissance, notre modèle est le seul à prédire que le salaire minimum préféré des travailleurs entrants est supérieur à celui des travailleurs installés. Ce résultat est intéressant pour au moins deux raisons. Tout d'abord, il signifie que le soutien politique pour un salaire minimum élevé dépasse probablement le nombre des travailleurs en emploi à faible qualification. D'autre part, il conduit à réévaluer les coûts et les bénéfices 
$\mathrm{du}$ salaire minimum pour les différentes catégories de travailleurs en tenant compte du rôle assuranciel du salaire minium et du statut des éventuels enfants sur le marché du travail $^{17}$. Ces développements suggèrent une explication relativement simple au fait que les étudiants constituaient les principaux opposants au smic-jeune proposé par le gouvernement Balladur en 1995. Ces étudiants disposaient pour la plupart d'une couverture assurancielle familiale contre le risque chômage accru associé à un salaire minimum élevé. Ils anticipaient donc une détérioration de leur bien-être.

Une dernière question concerne les effets du facteur d'altruisme sur le salaire minimum voté, l'offre de travail des vieux et le chômage des jeunes. A cette fin, nous identifions le taux de chômage d'équilibre des jeunes effectif au taux de chômage désiré par les travailleurs âgés.

proposition 6 (i) $\widehat{u}^{j}$ ne dépend pas de $\beta$

(ii) $\widehat{u}^{v}$ est une fonction croissante de $\beta$

(iii) $l^{0}$ et $l^{1}$ sont des fonctions croissantes de $\beta$

Le taux de chômage désiré par les jeunes est indépendant du facteur d'altruisme. En revanche, le taux de chômage désiré par les vieux en est une fonction croissante. La raison est la suivante: plus le facteur d'altruisme est élevé, plus les préférences des vieux reflètent celle de leurs enfants. Or, la proposition 5 montre que le taux de chômage désiré par les vieux est inférieur au taux de chômage préféré des jeunes. Cet écart se résorbe à mesure que le facteur d'altruisme s'accroît. La proposition 6 nous apprend également que cette augmentation du chômage des jeunes traduit l'augmentation simultanée du salaire minimum et de l'offre de travail des vieux. De ce point de vue, l'endogénéisation du salaire minimum ne fait que renforcer les mécanismes mis en exergue en section 3 lorsque le salaire minimum était exogène.

\section{Conclusion}

Cette étude part des faits stylisés suivants. Les jeunes connaissent des taux de chômage bien plus importants que les autres générations; il est communément admis que ces difficultés trouvent leur origine dans le fonctionnement du marché du travail, privilégiant les travailleurs installés plus âgés au détriment des entrants plus jeunes. D'autre part, ces mêmes travailleurs installés compensent au sein de la famille les conséquences de ce fonctionnement, notamment au travers de transferts inter vivo massifs. Pour certains,

\footnotetext{
${ }^{17}$ Une fois ces statuts pris en compte, il est possible de renverser la hiérarchie usuelle : les principaux bénéficiaires du salaire minimum pouvant être les jeunes plutôt que leurs parents. La démarche pourrait être étendue au cas du conjoint.
} 
le second phénomène ne serait qu'une conséquence du premier. Nous adoptons une démarche résolument plus polémique: le but louable de fournir le niveau de vie le plus élevé possible à leurs enfants pousse les parents à obtenir des revenus salariaux incompatibles avec le plein emploi des jeunes. L'altruisme parental serait ainsi aux sources des difficultés rencontrées par les jeunes sur le marché du travail.

Cet argument prend corps dans un modèle à générations imbriquées où les individus vivent et travaillent deux périodes. Les parents valorisent l'utilité de leurs enfants, et choisissent l'intensité de leur offre de travail; travail des enfants et travail des parents sont parfaitement substituables dans la production. L'offre de travail des parents augmente avec le facteur d'altruisme; cette augmentation induit une baisse de salaire horaire, ou une augmentation du chômage des jeunes lorsque le salaire minimum sature. Ce mécanisme donne son titre à ce travail; il est suivi de deux extensions. Dans la première, la productivité des travailleurs âgés est négativement reliée à la durée de chômage passée. Cette hypothèse donne lieu à des cycles de période deux, où une situation de faible chômage, faible offre de travail alterne avec une situation de fort chômage, forte offre de travail. Dans la seconde, nous étudions le support politique pour le salaire minimum lorsque la productivité des jeunes est soumise à un aléa. Lorsque l'altruisme est opérant, le salaire minimum préféré des jeunes est supérieur à celui des vieux. Une hausse du facteur d'altruisme tend à augmenter le salaire minimum, le chômage des jeunes et l'offre de travail des vieux.

Il est tentant d'utiliser ce canevas théorique pour en déduire une grille de lecture de l'hétérogénéité des performances des marchés du travail des pays développés. Une telle démarche nous semble vaine: notre modèle est beaucoup trop abstrait, et les causes ultimes de sous-emploi trop évidentes. Les américains n'aiment pas nécessairement moins leurs enfants que les français! 


\section{ANNEXES}

\section{A Démonstrations}

proposition 1 La contrainte $l_{t+1} \leq 1$ est toujours vérifiée puisque $U_{3} \rightarrow \infty$ lorsque $\ell_{t+1} \rightarrow 0$. Quant à la contrainte $l_{t+1} \geq 0$, elle est nécessairement satisfaite dès lors que la contrainte $x_{t+1} \geq 0$ tient. En effet, (2) implique que $d_{t+1}<-x_{t+1}$ quand $l_{t+1}<0$, de sorte que $d_{t+1}<0$ quand $x_{t+1}<0$, ce qui est impossible puisque $U_{2} \rightarrow \infty$ lorsque $d_{t+1} \rightarrow 0$. Il suffit donc de maximiser (3) sous la contrainte $x_{t+1} \geq 0$.

L'objectif est strictement concave en $l_{t+1}$ et $x_{t+1}$. Les conditions du premier ordre définissent donc un maximimum. Si $\Delta_{t+1} \geq 0$, alors $x() \geq$.0 et (ii) est vraie. Si $\Delta_{t+1}<0$, alors la contrainte $x_{t+1} \geq 0$ sature. L'offre de travail résulte ainsi de

$$
\max \left\{\alpha \ln l_{t+1}+\gamma \ln \left(1-l_{t+1}\right)\right\}
$$

ce qui donne (i).

proposition 2 (i) Si $\beta a \geq \alpha+\gamma$, la contrainte $x_{t+1} \geq 0$ ne sature jamais. Pour $w_{\min }=$ $w_{\max }, u_{n c}=1$, alors que pour $w_{\min } \leq \widehat{w}^{n c}, w^{*}=\widehat{w}^{n c}$ et $u^{*}=0$. Le résultat s'en suit de la continuité et de la stricte monotonicité de $u_{n c}$ par rapport à $w_{\min }$.

(ii) Soit $\bar{u}=1-\frac{\beta}{\alpha+\gamma} a$. Si $\beta a<\alpha+\gamma$, la contrainte $x_{t+1} \geq 0$ sature tant que $u<\bar{u}$, ce qui est équivalent à $u_{n c}=u_{c}$. Le résultat s'en suit de la continuité et de la stricte monotonicité des fonctions $u_{c}$ et $u_{n c}$ par rapport à $w_{\text {min }}$.

proposition 3 (i) On sait que

$$
\widehat{w}=F^{\prime}(1+a \widetilde{l}(a, 0))
$$

Or $\widetilde{l}(a, 0) \leq l^{n c}(\beta, 0)<1$. Donc $\widehat{w}>F^{\prime}(1+a)>w_{\min }$. Il suit que $u^{*}=0$ pour tout $\beta \geq 0$. D'autre part, la proposition 1 énonce que $l^{*}=l^{n c}$ si seulement si $\Delta \geq 0$, c'est-à-dire si et seulement si $\beta \geq \beta_{1}$. Le point (i) suit.

(ii) Comme $1+a l^{c}=1+\alpha a /(\alpha+\gamma)$, on a $\widehat{w}^{c} \geq w_{\min }$. Donc $u^{*}=0$ et $l^{*}=l^{c}$ si $\beta<\beta_{1}$. Lorsque $\beta \geq \beta_{1}, \Delta \geq 0$ et $l^{*}=l^{n c}\left(\beta, u^{*}\right)$. Tant que $\beta<\beta_{2}$, $\widehat{w}^{n c}>w_{\text {min }}$ et $u^{*}=0$. En revanche, si $\beta \geq \beta_{2}$ alors $\widehat{w}^{n c} \leq w_{\min }$ et $u^{*}=u^{n c}(\beta)$. Ceci clôt la démonstration de (ii).

(iii) Comme $1+a \widetilde{l}(a, 0) \geq 1+\alpha a /(\alpha+\gamma)$, $\widehat{w} \leq w_{\text {min }}$ et $u^{*} \geq 0$, ces deux dernières inégalités étant strictes lorsque $w_{\min }>F^{\prime}(1+\alpha a /(\alpha+\gamma))$. Ainsi, l'une des deux situations suivantes prévaut: ou $u^{*}=u^{c}$, ou $u^{*}=u^{n c}$. Nous montrons que $u^{*}=u^{n c}$ si et seulement si $\beta \geq \beta_{3}$. 
Commençons par la condition suffisante. Supposons $u^{*}=u^{n c}$. Alors

$$
\Delta=(\alpha+\beta+\gamma)\left[1-\frac{\alpha+\gamma}{\alpha+\beta} F^{\prime-1}\left(w_{\min }\right)\right]
$$

Or, $u^{*}=u^{n c}$ implique également que $\Delta \geq 0$, c'est-à-dire que $\beta \geq \beta_{3}$.

Pour établir la condition nécessaire $\left(\beta \geq \beta_{3} \Rightarrow u^{*}=u^{n c}\right)$, nous supposons que $u^{*} \neq u^{n c}$. Donc, $u^{*}=u^{c}$. Ainsi, $\Delta=(\beta+\alpha) a-(\alpha+\gamma) F^{\prime-1}\left(w_{\min }\right)$. Or, $u^{*}=u^{c}$ implique également que $\Delta<0$. Par conséquent, $\beta<\beta_{3}$. On en déduit que $\beta \geq \beta_{3}$ implique $u^{*}=u^{n c}$.

proposition 4 Soit la fonction $Z:[0,1] \rightarrow I \subset[0,1]$ telle que

$$
Z(u)=\max \langle\min \{\psi(u), 1\}, 0\rangle
$$

La dynamique s'écrit:

$$
u_{t}=Z\left(u_{t-1}\right)
$$

Comme la fonction $Z$ est décroissante, il existe un unique état stationnaire $u^{*}$. Soit alors la fonction $\phi=Z \circ Z$, fonction croissante de [0,1] dans [0,1]. Soit également la suite $x_{t}$ définie par

$$
\begin{aligned}
x_{t+1} & =\phi\left(x_{t}\right) \\
x_{0} & =u_{0}
\end{aligned}
$$

Comme $\phi$ est croissante, $\left\{x_{t}\right\}$ suit une dynamique monotone. Comme $x_{t}$ est bornée, elle converge vers un état stationnaire $\bar{x}$. Deux cas sont alors possibles:

- cas 1: $\bar{x}=u^{*}$, alors $u_{t}$ tend vers $u^{*}$ lorsque $t$ vers l'infini;

- cas 2: $\bar{x} \neq u^{*}$, alors $u_{t}$ converge vers le cycle de période $2\{\bar{x}, Z(\bar{x})\}$.

proposition 5 (i). Le bien-être des propriétéaires terriens se confond avec le revenu engendré par le facteur terre. Dans la mesure où la technologie est Cobb-Douglas, ce revenu est une fonction strictement décroissante du taux de chômage des jeunes. Ainsi, $\widehat{u}^{r}=0$.

(ii). Soit les fonctions $\phi_{j}$ et $\phi_{v}$ telles que

$$
\begin{aligned}
\phi_{j}(u) & =(\sigma-1) \ln [a+(b p+1-p)(1-u)] \\
& +p \ln [a+b(1-u)]+(1-p) \ln [a+1-u] \\
\phi_{v}(u) & =\phi_{j}(u)+\frac{\gamma}{\alpha+\beta}\{p \ln [a+b(1-u)]+(1-p) \ln [a+1-u]\}
\end{aligned}
$$


Jeunes comme vieux maximisent leur espérance d'utilité, données respectivement par $Z \equiv E z^{i}$ et $V \equiv E v^{i}$. A partir de (33) et (34), il vient:

$$
\begin{aligned}
Z & =\ln w+p \ln (a+(1-u) b)+(1-p) \ln (a+1-u)+c t e \\
V & =(\alpha+\beta) Z+\gamma\{p \ln [a+b(1-u)]+(1-p) \ln [a+1-u]\}
\end{aligned}
$$

Or,

$$
\ln w=(\sigma-1) \ln [a+(b p+1-p)(1-u)]+c t e
$$

On en déduit que

$$
\widehat{u}^{i} \in \arg \max _{u \in[0,1]} \phi_{i}(u), i=j, v
$$

Les fonctions $\phi_{j}$ et $\phi_{v}$ sont continûement dérivables, avec

$$
\begin{aligned}
& \phi_{j}(0)=(\sigma-1) \ln [a+b p+1-p]+p \ln [a+b]+(1-p) \ln [a+1] \\
& \phi_{j}(1)=\sigma \ln a>0 \\
& \phi_{v}(0)=\phi_{j}(0)+\frac{\gamma}{\alpha+\beta}\{p \ln [a+b]+(1-p) \ln [a+1]\} \\
& \phi_{v}(1)=\phi_{j}(1)+\frac{\gamma}{\alpha+\beta} \ln a>\phi_{j}(1)>0
\end{aligned}
$$

D'autre part, $\phi_{j}^{\prime}(u)>\phi_{v}^{\prime}(u)$ pour $u \in(0,1)$. Or, $\phi_{j}^{\prime}(u)$ est du signe de

$$
P_{j}(u)=A_{0}+A_{1} u+A_{2} u^{2}
$$

où

$$
\begin{aligned}
A_{0} & =(b p+1-p)[(1-\sigma)(a+b)(a+1)-b p(a+1)-(1-p)(a+b)] \\
& -b p a(a+1)-(1-p) a(a+b) \\
A_{1} & =a\{(b p+1-p)[b(p+\sigma-1)-p+\sigma]+b\} \\
A_{2} & =-\sigma b(b p+1-p)
\end{aligned}
$$

Comme $P_{j}(1)=-\sigma(b p+1-p)<0, \widehat{u}^{j}<1$. Trois cas sont envisageables.

- cas 1: $A_{0}>0$. $P_{j}$ admet alors une unique racine sur $[0,1], u_{1}$, et $\widehat{u}^{j}=u_{1}$. Comme $\phi_{v}^{\prime}(u)<\phi_{j}^{\prime}(u)$ pour tout $\left.u \in\right] 0,1\left[, \phi_{v}^{\prime}(u)<0\right.$ pour tout $u \geq u_{1}$ et $\widehat{u}^{j}>\widehat{u}^{v}$.

- cas 2: $A_{0}<0$ et $P_{j}$ n'a pas de racine sur ]0,1[. Alors $\widehat{u}^{j}=0$. Comme $\phi_{v}^{\prime}(u) \leq$ $\phi_{j}^{\prime}(u)$ pour tout $u \in[0,1], \widehat{u}^{v}=\widehat{u}^{j}=0$.

- cas 3: $A_{0}<0$ et $P_{j}$ admet deux racines sur $] 0,1\left[, u_{1}\right.$ et $u_{2}$. Alors ${ }^{18}$

$$
\widehat{u}^{j}=\left\{\begin{array}{l}
0 \text { si } \phi_{j}\left(u_{2}\right)-\phi_{j}(0) \leq 0 \\
u_{2} \text { sinon }
\end{array}\right.
$$

\footnotetext{
${ }^{18}$ Nous supposons arbitrairement que $\widehat{u}^{j}=0$ lorsque $\phi_{j}\left(u_{2}\right)=\phi_{j}(0)$.
} 
Comme $\phi_{v}^{\prime}(u)<\phi_{j}^{\prime}(u)$ pour tout $\left.u \in\right] 0,1\left[\right.$, ou $\phi_{v}^{\prime}$ admet deux racines sur ]0, 1 , ou elle n'en a pas. Si elle n'en a pas, $\widehat{u}^{v}=0 \leq \widehat{u}^{j}$. Si elle en a deux, $u_{3}$ et $u_{4}<u_{2}$, alors

$$
\widehat{u}^{v}=\left\{\begin{array}{l}
0 \text { si } \phi_{v}\left(u_{4}\right)-\phi_{v}(0) \leq 0 \\
u_{4} \text { sinon }
\end{array}\right.
$$

Or,

$$
\begin{aligned}
\phi_{v}\left(u_{4}\right)-\phi_{v}(0) & =\int_{0}^{u_{4}} \phi_{v}^{\prime}(u) d u \\
\phi_{j}\left(u_{2}\right)-\phi_{j}(0) & =\int_{0}^{u_{2}} \phi_{j}^{\prime}(u) d u
\end{aligned}
$$

Donc

$$
\begin{aligned}
\phi_{v}\left(u_{4}\right)-\phi_{v}(0) & <\int_{0}^{u_{4}} \phi_{j}^{\prime}(u) d u \\
& <\int_{0}^{u_{2}} \phi_{j}^{\prime}(u) d u=\phi_{j}\left(u_{2}\right)-\phi_{j}(0)
\end{aligned}
$$

Par conséquent, $\widehat{u}^{v}=u_{4}>0$ seulement si $\widehat{u}^{j}=u_{2}>u_{4}$. Le résultat (i) se déduit des cas 1,2 et 3 .

(iii). Nous montrons que $\widehat{u}^{j}>0$ dès lors que $\sigma$ est suffisamment faible. Le résultat s'en suit dès lors que $\widehat{u}^{v}$ tend vers $\widehat{u}^{j}$ lorsque $\gamma$ tend vers 0 . Il nous suffit de montrer que $\phi_{j}^{\prime}(0)>0$ lorsque $\sigma$ tend vers 0 . Or, $\phi_{j}^{\prime}(0)$ est du signe de $A_{0}$. Il vient

$$
\lim _{\sigma \rightarrow 0} A_{0}=a p(1-p)(1-b)^{2}>0
$$

proposition 6 (i) Au cours de la preuve de la proposition 6, nous montrons que

$$
\widehat{u}^{i} \in \arg \max _{u \in[0,1]} \phi_{i}(u), i=j, v
$$

Par conséquent, $\widehat{u}^{j}$ est indépendant de $\beta$ puisque $\phi_{j}$ est elle-même indépendante de $\beta$.

(ii) Le résultat est trivial lorsque $\widehat{u}^{v}=0$. Si $\widehat{u}^{v}>0$, il résulte nécessairement de

$$
\frac{\partial \phi^{v}\left(\widehat{u}^{v}, \beta\right)}{\partial \beta}=0
$$

Le théorême des fonctions implicites implique que

$$
\frac{\partial^{2} \phi^{v}\left(\widehat{u}^{v}, \beta\right)}{\partial u^{2}} \frac{d \widehat{u}^{v}}{d \beta}+\frac{\partial^{2} \phi^{v}\left(\widehat{u}^{v}, \beta\right)}{\partial u \partial \beta}=0
$$

Or, $\partial^{2} \phi^{v}\left(\widehat{u}^{v}, \beta\right) / \partial u^{2}<0$ car $\widehat{u}^{v}$ est un maximum. Il s'en suit que $d \widehat{u}^{v} / d \beta>0$ si et seulement si $\partial^{2} \phi^{v}\left(\widehat{u}^{v}, \beta\right) /(\partial u \partial \beta)>0$. Il vient

$$
\partial^{2} \phi^{v}\left(\widehat{u}^{v}, \beta\right) /(\partial u \partial \beta)=\frac{\gamma}{(\alpha+\beta)^{2}}\left[\frac{p b}{a+b(1-u)}+\frac{1-p}{a+1-u}\right]>0
$$


(iii) Rappelons que par hypothèse le taux de chômage effectif $u^{*}$ reflète les préférences des travailleurs âgés (les électeurs médians). Ainsi,

$$
\frac{d l^{i}}{d \beta}=\frac{\gamma}{(\alpha+\beta+\gamma)^{2}}\left\{1+\frac{b^{i}}{a}\left[(\alpha+\beta+\gamma) \frac{d u^{*}}{d \beta}+1-u^{*}\right]\right\}>0
$$

$\operatorname{car} d u^{*} / d \beta \geq 0$.

\section{References}

[1] Abowd J., F. Kramarz, D. Margolis et T. Philippon, 2001, The tail of two countries: minimum wage and employment in France and the United-States, working paper no 203, IZA.

[2] Agell J., 1999, On the benefits from rigid labour markets: norms, market failures, and social insurance, Economic Journal, 109, pp. F143-F164.

[3] Barro R., 1974, Are government bonds net wealth?, Journal of Political Economy, 82, pp. 1095-1117.

[4] Bazen S. et N. Skourias, 1997, Is there a negative effect of minimum wages on youth employment in France?, European Economic Review, 41, pp. 723-32.

[5] Becker S., S. Bentolila, A. Fernandes et A. Ichino, 2002, Job insecurity and children's emancipation: the Italian puzzle, Mimeo.

[6] Buchanan J., 1975, The samaritan's dilemma, in E. Phelps (éd.), Altruism, morality and economic theory, New York: Russell Sage Foundation.

[7] Boadway R. et N. Marceau, 1994, Minimum wage legislation and unemployment insurance as instruments for redistribution, Scandinavian Journal of Economics, 96, pp. $67-81$.

[8] Cahuc P. et H. Kempf, 2000, L'altruisme est-il socialement efficace ?, Revue du MAUSS, $\mathrm{N}^{\circ} 15$, pp. 223-246.

[9] Cahuc P. et P. Michel, 1996, Minimum wage, unemployment and growth, European Economic Review, 47, pp. 1463-1482.

[10] Coate S., 1995, Altruism, the samaritan's dilemma, and government transfer policy, American Economic Review, 85, pp. 46-57. 
[11] Decreuse B. et B. Wigniolle, 2002, Intergenerational conflicts and the minimum wage legislation, Cahier de la MSE.

[12] Diamond P., 1965, National debt in a neoclassical growth model, American Economic Review, 55, pp. 1126-1150.

[13] Dolado J., F. Kramarz, S. Machin, A. Manning, D. Margolis et C. Teulings, 1996, The economic impact of minimum wages in Europe, Economic Policy, 23, pp. 317-57.

[14] European Communities, 1999, Demographic statistics: data 1995-98, Luxembourg, Office for official publications of the European Community.

[15] Hu S.C., 1979, Social security, the supply of labor and capital accumulation, American Economic Review, 69, pp. 274-284.

[16] Lambrecht S., 2003, Altruistic bequests and non-negative savings, Louvain Economic Review, 69, pp. 349-470.

[17] Lambrecht S., P. Michel et J-P. Vidal, 2005, Public pensions and growth, European Economic Review, à paraître.

[18] Lindbeck A. et D. Snower, 1988, The insider outsider theory of employment and unemployment, MIT Press: Cambridge, MA.

[19] Michel P. et P. Pestieau, 2000, Retraite par répartition et âge de la retraite, Revue Economique, 51, pp. 15-30.

[20] Saint-Paul G., 1993, On the political economy of labour market flexibility, NBER Macroeconomics Annual, pp. 151-195.

[21] Saint-Paul G., 2001, Political economy of the labour market, Oxford and New York: Oxford University Press.

[22] Sobel R., 1999, Theory and evidence on the political economy of the minimum wage, Journal of Political Economy, 107, pp. 761-785.

[23] Stark O., 1989, Altruism and the quality of life, American Economic Review, vol. 79, pp. 86-90.

[24] Stigler G., 1946, The Economics of minimum wage legislation, American Economic Review, 36, pp. 358-365.

[25] Thibault E., 2002, Les déterminants économiques de l'émergence d'une classe de rentiers, Recherches Economiques de Louvain, 68, pp. 457-480. 
[26] Thibault E., 2004, Bequests and the intergenerational degree of altruism, Louvain Economic Review, 67, pp. 131-138. 